JONATHAN REYES GONZÁLEZ

IRENE REIG ALBEROLA

AITOR DEZA ATUTXA

ANTONIO MOYA LATORRE

RUBÉN TORMO GÓMEZ
Escuela de Arquitectura de Valencia

Universidad Politécnica de Valencia

info@carpevia.org

\title{
Ludocracia
}

\section{El juego como herramienta de revitalización urbana en la ciudad interfaz}

vol 12 / Jun.2015 127-144 pp Recibido: 23-11-2014 - revisado 12-12-2014 - aceptado: 02-02-2015 


\section{LUDOCRACY}

PLAY AS TOOL FOR URBAN REVIVAL AT THE INTERFACE CITY

\section{ABSTRACT}

We live in the city of fear. The excessive regulation and classification of what happens in our environment has pushed us into restricted rules of the game, in which there is no space left for improvisation, imagination or emotions. In this planned and managed environment by a few ones, the erroneous incorporation of the technology has increased the degree of control of the measurable variables, basing on them their public policy. This lack of care for intangible variables representing the human life of cities has caused, together with the introduction of the ICTs, the step towards the digital: a virtual place to achieve freedom and the debate that has been lost in the physical public space. Given this new physical-digital public space duality we consider its possible connection with the city as interface. The gamification of the processes of cities, the physical-digital hybridization of the urban games or the Lighter \& Quicker $\&$ Cheaper concept are present in contemporary innovative projects reviewed below. As a proposal for the future we bet on ludocracy as an actuator mechanism for the reconquest of the physical public space showing the city as an augmented playground.

\section{Keywords}

Physico-digital hybridization, augmented playground, interface city, gamitification, sentient city.

\section{RESUMEN}

Vivimos en la ciudad del miedo. La excesiva regulación y la categorización de lo que sucede en nuestro entorno nos han ceñido a unas reglas del juego limitadas, en las que queda poco margen para la improvisación, la imaginación y las emociones. La errónea incorporación de las tecnologías ha aumentado el grado de control que se tiene sobre variables medibles basando en ellas sus políticas públicas. Esta despreocupación por las variables intangibles que representan la vida humana de las ciudades ha provocado, junto a la introducción de las TICs en nuestra rutina, el traslado del espacio público a la esfera digital: un espacio virtual donde obtener la libertad y recuperar el debate que se ha perdido en el espacio público físico. Ante esta nueva dualidad físico-digital del espacio público nos planteamos la posible conexión de ambos con la ciudad como interfaz. La ludificación de los procesos de las ciudades, la hibridación físico-digital en juegos urbanos o el concepto Lighter \& Quicker \& Cheaper están presentes en proyectos innovadores contemporáneos reseñados más adelante. Como propuesta de futuro apostaremos por la ludocracia que se sirve del juego como mecanismo accionador para la reconquista del espacio público físico mostrando la ciudad como un tablero de juego aumentado.

\section{Palabras Clave}

Hibridación físico-digital, espacio de juego ampliado, ciudad interfaz, ludificación, ciudad sensible. 


\section{PRÓLOGO. SITUACIÓN PROBLEMÁTICA ACTUAL: LA CIUDAD DEL MIEDO}

La ciudad contemporánea es una ciudad asustada y atemorizada. La utopía de una sociedad segura y perfecta, desde el punto de vista de una rutina clara, organizada e invariable, nos ha llevado a perseguir y a tratar de erradicar cualquier tipo de riesgo en el entorno urbano:

Esta obsesión por la seguridad está dejando cada vez trazos más visibles en nuestro espacio público y en el modo en que organizamos nuestras ciudades: resaltes para controlar la velocidad, zonas libres de fumadores y cámaras de vigilancia sirven a una sociedad que vive en la prevención (Baber et al., 2005; p. 25).

Este sentimiento permanece arraigado en nuestra cultura y aparece, tal y como sugiere Bert de Muynck, como una de las fuerzas directoras que se esconden tras la reorganización del espacio público en la ciudad contemporánea, prescribiendo un entorno cada vez más yermo y neutro donde lo excepcional e improvisado es percibido como amenaza que debe ser erradicada. El control, la vigilancia y la represión son factores que quedan así justificados y priorizados a la hora de hacer ciudad. "El problema individual del hombre occidental (ansiedad, seguridad, cultura, consumo, velocidad) salta así al espacio público, y se convierte en un problema global de la ciudad y su espacio público" (op. cit.).

Los trazos del miedo en la ciudad contemporánea han venido dibujándose en la historia del urbanismo. Murallas, fortalezas y atalayas aparecen como trazos históricos de una cultura del miedo sobre la que se han construido nuestras ciudades. El planeamiento urbano aparece así en muchas ocasiones a lo largo de nuestra historia como una herramienta de control en manos de un poder político con propósitos económicos y políticos muy concretos. Muchos de los grandes planes urbanos que han transformado nuestras ciudades durante los últimos siglos sirvieron como instrumento de regulación y contención de los grandes movimientos revolucionarios de las clases rebeldes. David Harvey (2013) nos recuerda así como el Plan Haussmann supuso "derribar los viejos barrios de París empleando poderes excepcionales de expropiación, supuestamente en beneficio público, en nombre de los derechos de ciudadanía, la restauración ambiental y la renovación urbana. Consiguió así deliberadamente expulsar del centro de París, junto con las industrias insalubres, a gran parte de la clase obrera y otros elementos rebeldes que constituían una amenaza para el orden público y por supuesto para el poder político. Creyendo (incorrectamente, como se comprobó en la Comuna revolucionaria de París de 1871) que aquella reforma urbana ofrecía un nivel suficiente de vigilancia y control militar como para asegurar el fácil sometimiento por la fuerza de las clases rebeldes." (op.cit.)

Se trata de un proceso que ha seguido y sigue reproduciéndose hoy en numerosas ciudades a lo largo y ancho del planeta. Los proyectos de Robert Moses en la ciudad de Nueva York, los actuales procesos urbanos que están teniendo lugar en parte de Asia -Bombay, Nueva Delhi o Seúl- o los planes para las favelas en Río de Janeiro son solo algunos de los casos que, desde la idea de progreso, bienestar y calidad públicos siguen en la actualidad fracturando nuestras ciudades a través del miedo y con intereses económicos como factores directores. La Calle de la Paz y la Avenida de Barón de Cárcer en Valencia son ejemplos a nivel local. Ambas fueron planificadas como dos brechas que atravesaran de lado a lado el casco antiguo de la ciudad, aunque finalmente no se completaron. Tampoco la prolongación de la avenida de Blasco Ibáñez hasta el mar, en la misma ciudad, ha podido prosperar gracias a la presión mediática y a la situación económica actual, que hace inviable una obra de tal envergadura. 
La arquitectura y el diseño urbano, tomados como respuesta a los problemas funcionales y técnicos, parecen haber olvidado su capacidad de hacer espacio público como espacio de interacción y construcción de cultura y ciudadanía. Se han convertido en instrumentos de organización y control de la ciudad, generando una trama urbana en la que el miedo no aparece como un elemento puntual, sino que es una parte intrínseca de su naturaleza.

El espacio público ha sido fragmentado para posteriormente ser vendido, clasificado y reorganizado en sus diferentes zonas hasta su control absoluto. Cualquier elemento que escape a la estricta diferenciación y clasificación que organizan lo urbano son interpretados como amenaza. Lo improvisado, lo complejo, la sorpresa y la "serendipia" son así elementos apartados del planeamiento urbano.

"Estos sentimientos no son sin embargo expulsados de la ciudad -hablando en términos urbanísticos-, pero sí de los edificios. El miedo se convierte así en el único factor que conecta los espacios privados neuróticos" (Baber et al., 2005; p. 25). "La ciudad deja de ser el seno de producción cultural y libre debate para convertirse en un espacio de control". Estamos, por tanto, ante un espacio público asustado y confiscado cuyas posibilidades de uso y desarrollo son definidas sólo por unos pocos. Este excesivo control y las limitaciones impuestas, acompañados de las pesadas gestiones burocráticas necesarias para cualquier tipo de intervención, limitan y apagan la vitalidad de nuestras calles y plazas, condenadas a ser espacios de mero tránsito y consumo (Borja, 1998). Resulta difícil jugar en una ciudad como esta.

\section{MARCO TEÓRICO: LA CIUDAD COMO INTERFAZ}

\subsection{Espacio público como germen de la ciudad}

El ser humano es social por naturaleza (Aristóteles, s.IV a.C.). Es a través de la interacción, la comparación y el contacto con el igual como se produce la maduración y el crecimiento de cada individuo y del conjunto de la sociedad (Tonucci, 2009). Es por tanto el espacio público el lugar de encuentro e intercambio donde se teje la ciudad, se construye la cultura común y donde nace la ciudadanía. El juego, entendido en su sentido más amplio -desde su capacidad de generar interacción entre personas y de éstas con su entorno- se convierte en la herramienta más rica para construir el espacio público como ese entorno abstracto de contacto y aprendizaje.

Sin embargo, tal y como hemos explicado, el espacio público es víctima de una excesiva fragmentación y de determinadas políticas sectoriales que limitan y coartan su vitalidad. Las actividades propias del espacio público se vuelcan progresivamente hacia lo digital. La calle ya no es más un espacio de interacción social y juego: ahora este se concentra en nuestras casas, en nuestros dispositivos móviles o videoconsolas.

Las posibilidades que nos ofrecen las nuevas tecnologías de comunicación nos invitan a refugiarnos en el hogar (Castells, 1997), y ante esta situación vemos cómo cada vez más el debate público se desplaza al espacio virtual y a entornos privados, abandonando el público. Y es que, si atendemos a la definición de espacio público como aquel espacio de propiedad pública, de dominio y uso público, parece que Internet se ha posicionado durante los últimos años como el espacio público por excelencia. Como explica di Siena (2009; p. 6), lo digital 
"parece ofrecer cada vez más un 'lugar' para las relaciones sociales alternativo a los lugares 'tradicionales'”, y la esfera pública se ve así cada vez más volcada hacia lo digital.

Este hecho puede ser interpretado como uno de los problemas causantes de incrementar el creciente vaciamiento y abandono del espacio público o, por el contrario, se puede considerar Internet como una extraordinaria herramienta capaz de desarrollar nuevas dinámicas de relación capaces de transformar el modo en que vivimos el entorno urbano. El espacio público sigue existiendo, la necesidad de encuentro, interacción y juego entre iguales perdura, pero se ha transformado porque el contexto es distinto:

Lo que está en crisis no es la utilización del espacio público en sí mismo, sino su comprensión reduccionista, así como la creencia de que los instrumentos que se desarrollaron en su seno entre el s XIX y la primera mitad del s. XX tienen la misma vigencia y validez en la actualidad. Son los usos y los usuarios los que cambian, pero la necesidad de encuentro en el espacio siempre ha estado y estará (di Siena, 2009; p. 48).

\subsection{Espacio público como germen de la ciudad}

Parece, por tanto, necesario y fundamental revisar los instrumentos y los procesos heredados del diseño y la gestión del espacio público para integrarlos y adaptarlos al contexto actual. Hoy el espacio público es un espacio híbrido que cuenta con una doble naturaleza física y digital.

En este contexto, hacer ciudad no puede reducirse a la definición física de esta, pues la ciudad es algo mucho más complejo y rico. La ciudad es, como dice Mark Sephard (2011), una condición de entorno donde reina la sorpresa y la serendipia.

Sin abandonar la idea de hardware a la hora de hacer ciudad es importante empezar a pensar también en términos de software. La integración de esta piel digital aumenta la experiencia de lo urbano y genera nuevas dinámicas de comunicación y relación con el entorno heredadas de los modelos de funcionamiento de la red (Sánchez, 2009).

En esta situación, el juego aparece como un motor que activa este sistema operativo urbano compuesto de hardware y software. Se sitúa como catalizador que promueve la interacción y la cooperación entre ciudadanos en el entorno urbano ahora ampliado a través de lo digital. Lo lúdico se convierte en una herramienta de aprendizaje social, sensorial y cultural que tiene como base la ciudad construida.

\subsection{Ciudad en código abierto}

La integración de nuevas tecnologías como herramientas capaces de aumentar la experiencia del juego en el entorno urbano debe ir necesariamente acompañada de un cambio de sensibilidad y protagonismo de sus usuarios. Así, el espacio público como escenario de juego no hereda únicamente los medios y las herramientas digitales, sino que adopta los modos organizativos y las dinámicas propias de la cultura en red y el software libre. Como afirma Reyes (2013; p. 60), "más allá de una sola implementación tecnológica, se trata de una transformación social 
en la que el ciudadano o el jugador percibe cada vez más su capacidad como agente activo de cambio".

El ciudadano o el jugador -ahora híbrido- en este contexto, ha dejado de ser un simple receptáculo pasivo de contenido, o un sujeto anónimo, para convertirse en un agente activo capaz de generar y compartir con otros usuarios nuevos contenidos y transformar su entorno inmediato a través del juego. Asistimos así a una superación de los tradicionales modelos organizativos piramidales y cerrados en favor de sistemas distribuidos, donde el juego se produce en código abierto para adoptar un sistema de funcionamiento parecido al que se utiliza en el desarrollo de software libre. El usuario deja de tener un papel pasivo como observador y consumidor, para convertirse en un protagonista activo que puede acceder al código fuente del juego, para ampliarlo, modificarlo y posteriormente compartirlo (Corsín, 2014).

\subsection{La ciudad como interfaz}

Desde esta nueva forma de entender el espacio público y el modo de intervenir en él, la ciudad se parece más a una conversación que a un objeto, y la interacción -y no la delineación- es por tanto el más importante de sus elementos.

Existen varias acepciones para el término "interfaz" (Del ingl. interface, superficie de contacto):

1. Conexión física y funcional entre dos aparatos o sistemas independientes.

2. Zona de comunicación o acción de un sistema sobre otro.

3. Dispositivo que conecta dos aparatos o circuitos.

4. Dispositivo capaz de transformar las señales emitidas por un aparato en señales comprensibles por otro.

Podemos afirmar que el espacio público, tal y como la entendemos, es una interfaz de contacto donde tiene lugar la conexión física y funcional entre dos o más medios o dispositivos de cualquier tipo o, como proponen Basar y Miessen (2009) una "simple y eficaz mediadora entre dos dominios relacionados, pero no conectados entre sí". De esta manera, entendemos hoy la ciudad como un sistema complejo de relaciones capaz de establecer esa zona de comunicación y acción entre múltiples sistemas:

- Interfaz social: escenario capaz de propiciar el encuentro entre ciudadanos y comunidades afines.

- Interfaz cultural: espacio de integración en intercambio de distintas culturas.

- Interfaz físico - digital: vínculo de la ciudad presencial con la digital. La información de interés relativa al espacio presencial es incorporada mediante la infraestructura digital.

Los distintos sistemas confluyen así, en un único espacio, interconectados de manera eficaz. El juego, como mecanismo accionador, se convierte en una herramienta desde la que experimentar, debatir, colaborar y construir nuestro entorno, que se manifiesta como una interfaz tangible. Ahora el espacio público es el soporte; y la calle, un tablero de juego aumentado. 
Como ya hemos comentado, muchas veces el diseño de nuestras ciudades limita lo extraordinario y lo fortuito, que al fin y al cabo son dos características propias del juego. De esta manera, la ciudad expulsa a la infancia, cuyo principal método para participar en la construcción en su entorno próximo es precisamente el juego. Si les negamos el derecho a jugar, como se hace de forma inconsciente en la ciudad del miedo, estamos negando la posibilidad de desarrollarse positivamente de forma física y mental; y de aprender valores como el respeto, la capacidad de liderazgo y la colaboración, defendidos y promovidos por Unicef (2004).

El colectivo de los niños aprende cuando algo de su entorno le emociona y le estimula, no con la repetición -como se creía en la revolución industrial, donde se obtenía una enseñanza estandarizada con el fin de que esos niños sirvieran a la sociedad- (Punset, 2012). En la experiencia urbana, los adultos se trasladan por la ciudad, mientras que los niños la atraviesan como si vivieran una sucesión de excitantes experiencias. Es por ello que algunos sociólogos apuestan por la re-infantilización "como mecanismo para restaurar una experiencia infantil de lo urbano: el amor por las esquinas, los quicios, los descampados, los escondites, los encuentros fortuitos, la dislocación de las funciones, el juego" (Delgado, 2011; p. 13); en definitiva, una ciudad más sensible (Figura 1 ).

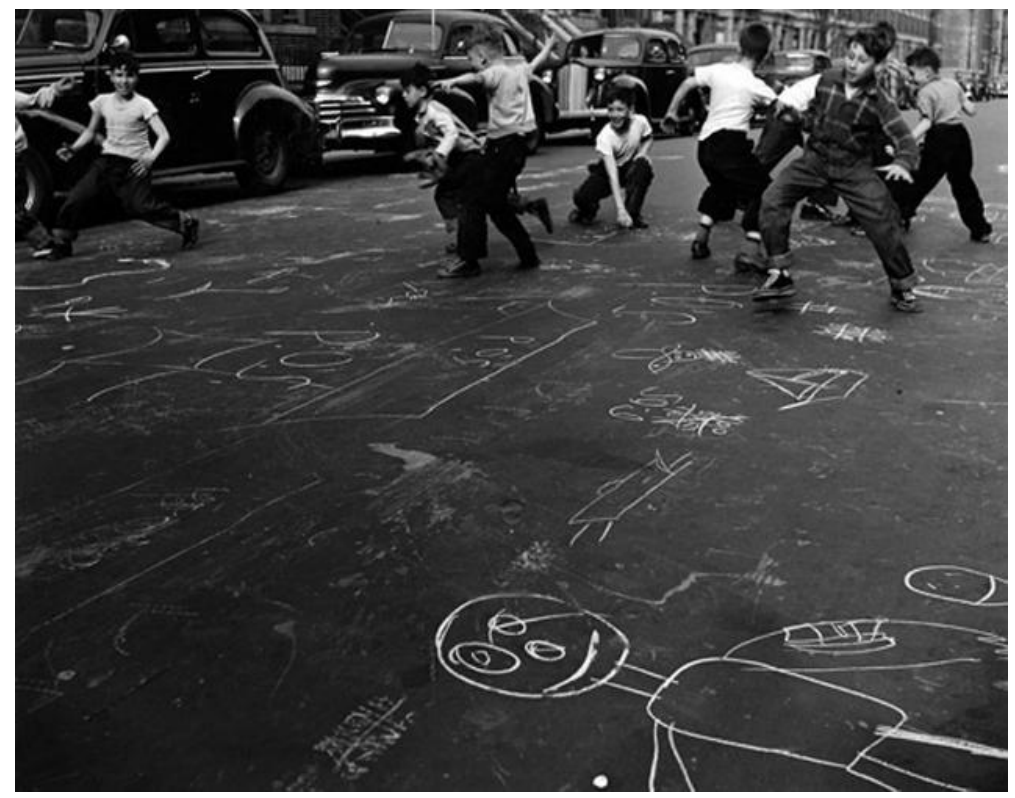

Figura 1. Arthur Leipzig, Chalk games, New York. 1959

Este comportamiento intuitivo, presente en el juego, puede tener varias lecturas dentro de las intervenciones urbanas. Por una parte, el juego puede ser una herramienta de conocimiento, aprendizaje, planificación y acercamiento de las necesidades del ciudadano para abordar un proyecto urbano de una manera más cercana, y a la vez ofrecer a la administración, a los diseñadores y a los demás agentes encargados de materializar un proyecto urbano la información necesaria para conocer las necesidades reales del entorno. 
Por otra parte, el juego en sí representa una acción de reivindicación y empoderamiento del espacio público como lugar libre y disfrutable por todos. Se trata de un desafío para las ciudades organizadas desde un planteamiento estricto. El traslado del juego a la calle vitaliza una ciudad. Para poder jugar en ella, la ciudad debe tener cierto carácter doméstico que posibilite la relación entre los ciudadanos y el uso libre del espacio público. El juego en los espacios públicos nos muestra el camino para superar de una manera innata, natural e inocente los miedos a la improvisación.

\subsection{Ludificación urbana}

La ludificación, en inglés gamification -término que se emplea para definir el uso de dinámicas propias de los juegos en actividades de por sí no lúdicas- (Deterding et al., 2011), se ha visto engrandecido en la última década como consecuencia del auge del entorno digital y de los videojuegos. Mediante esta práctica se consigue que las personas intervengan dentro de actividades a priori aburridas, como puede ser conocer datos urbanísticos para participar en una intervención urbana, con una actitud más propositiva. Pues las características de sociabilidad, identificación con el lugar y cooperación son innatas a este tipo de procesos de aprendizaje.

El estudio Play the City (Figura 2), ubicado en Ámsterdam, es uno de los pioneros en la aplicación de este concepto. A través de los juegos, tanto físicos como digitales, consiguen conectar a agentes que se interesan en los procesos de transformación de la ciudad, generando una plataforma de inteligencia colectiva que permite evolucionar la ciudad teniendo presente una visión más integradora que los procesos urbanísticos tradicionales: todos los jugadores evolucionan en los futuros escenarios de negociación y colaboración, así como también entran en conflictos los unos con los otros. El juego se convierte en una herramienta de mediación que sobrepasa las limitaciones de lenguaje que pueden existir entre los diferentes agentes y ayuda a visualizar, mediante la transcripción de los datos obtenidos, las soluciones más valoradas.

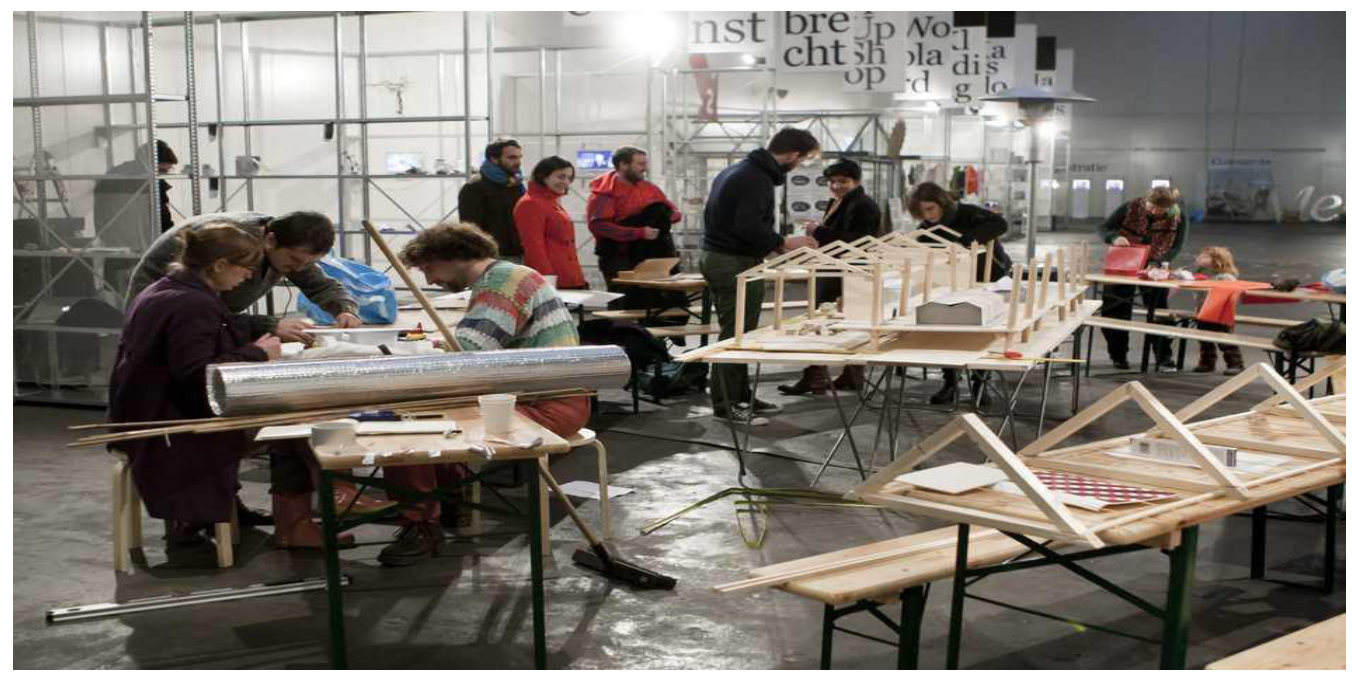

Figura 2. Preparing the city game. Proyecto: "Play van Gendthallen”. Play the City. 2013 
Otro tipo de experiencias más ligadas al arte, como Helium, de Félix Ríos, pretenden que reflexionemos de manera grupal sobre los problemas que acontecen en nuestras ciudades. Helium recrea una historia ficticia, donde la tierra es invadida por extraterrestres con los que debemos convivir. Se pretende generar una realidad paralela para reflexionar de manera colectiva entre todos los jugadores, sobre cuestiones políticas, sociales y económicas de nuestras ciudades: Helium busca dinamizar los procesos de discusión creativa sobre nuestras ciudades, registrar las diferencias culturales entre diversos grupos, ciudades y países, así como experimentar con la web como medio documental (Ríos, 2014).

\subsection{Hibridación de los juegos urbanos}

En la sociedad occidental desarrollada, donde el espacio público se ha rendido a las estrictas normas de funcionamiento y la introducción de las tecnologías por la obsesión del control de datos, Internet y los videojuegos han atraído a los más pequeños -y también, a los menos- de vuelta a casa. Para recuperar el espacio público como lugar para el juego debemos conseguir que la calle se convierta en grandes salas de juego y aventura. Es necesario olvidar que el juego es una cosa de niños y empezar a dejarnos sorprender por lo extraordinario que puede pasar en nuestras ciudades (Infans' Fans, 2014).

Hybrid Play es un proyecto liderado por Clara Boj y Diego Díaz con el que se pretende transformar los espacios de juego físicos en videojuegos. Mediante sensores que se acoplan a los parques infantiles ya existentes, que se conectan el movimiento real de los jugadores con el de los personajes virtuales que habitan en la APP. Los beneficios de la propuesta son claros: además de promover el juego en el exterior, la vida activa y las relaciones sociales, su planteamiento desde el código abierto favorece la personalización y el libre acceso (Hybrid Play, 2014) (Figura 3).

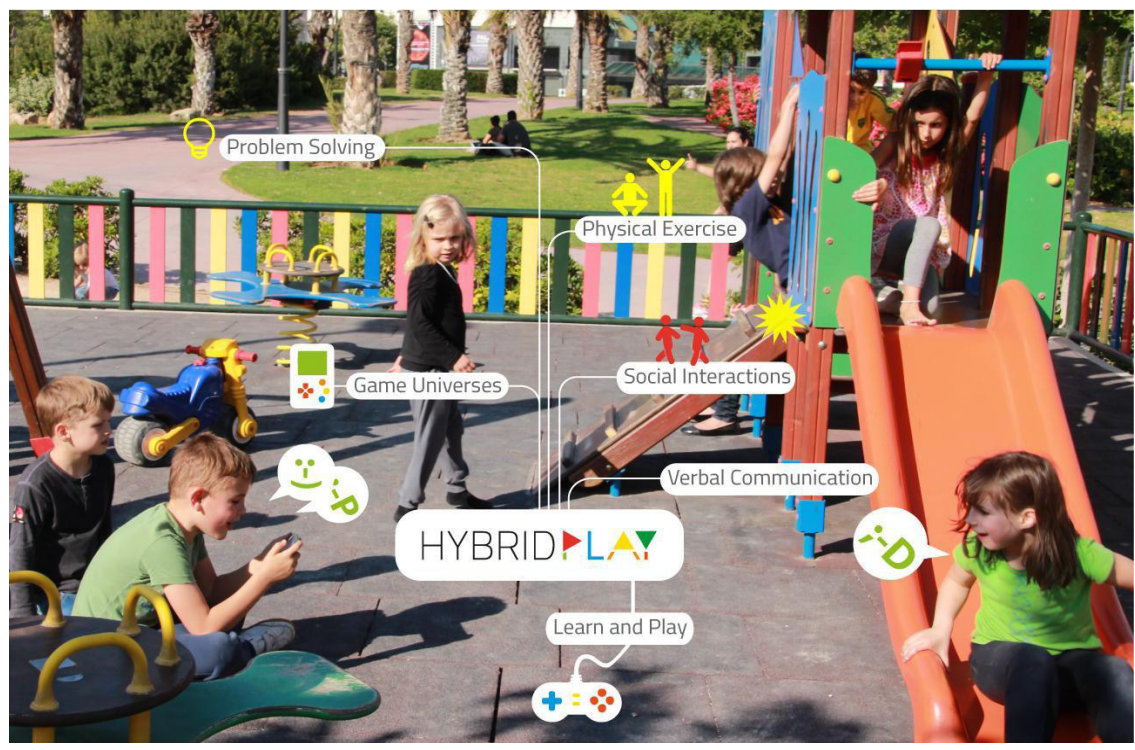

Figura 3. Hybrid Play. Clara Boj y Diego Díaz. 2014 
En Carpe Via, entendemos el juego urbano como una forma de reivindicación del espacio público como plataforma para el juego, una herramienta capaz de fomentar la identidad local a partir de la tradición y la cultura; un soporte sobre el que experimentar nuevas formas de uso de la tecnología, así como una oportunidad de llevar a cabo proyectos de diseño abierto, donde los propios jugadores pueden replicar, ampliar y modificar. A través de la capa digital conseguimos ubicarnos en la realidad glocal -modo de vida en el espacio local condicionado por nuestra exigencia de comunicarnos con personas que viven en dimensión global (di Siena, 2009), permitiendo que otras personas no presentes en el entorno local puedan enriquecerse y colaborar dentro del proyecto.

Entendemos que los juegos urbanos no suponen únicamente una manera de intervención lowcost para reactivar la ciudad y atraer de nuevo a los ciudadanos a la calle, sino que se trata también de una manera de tantear el terreno, de conocer las posibilidades y de actuar en el entorno construido sin tener que realizar grandes esfuerzos económicos -al igual que otras acciones de placemaking que cumplen con la filosofía lighter \& quicker \& cheaper-. Éstas, se centran en convertir un espacio físico en un lugar que contempla la dimensión emocional, de vínculo entre las personas y su entorno (Project for Public Spaces). Más allá de la transformación puntual que se pueda llevar a cabo tras implantar un juego urbano, se incide en la concienciación colectiva de los usos que se han perdido y se pueden recuperar y adaptar al espacio público, como el juego (Navazo, 2013).

En España se ha llevado a cabo Play Day, una propuesta del equipo Zaramari en Bilbao para recuperar durante un día el juego en la calle como herramienta para la socialización y la reactivación urbana. La instalación temporal de los juegos en un contexto ayuda a imaginar una calle diferente, con más creatividad y menos tránsito.

Se trata, pues, de un tipo de intervenciones sobre una ciudad heredada que contrasta con los métodos tradicionales de resolución de problemas para la reactivación de espacios públicos degradados y que requiere pequeñas cantidades de dinero, tiempo y energía. Estas intervenciones surten efecto desde el primer momento que son llevadas a cabo. Gracias a su reversibilidad, se puede empezar fácilmente una nueva versión de prueba que, en el caso de ser aceptada y demandada, pueda tomar una forma permanente. El juego se constituye así como un catalizador de debates entre ciudadanos.

\section{EXPERIENCIAS PROPIAS: JUGANDO CON LA CALLE}

Nuestras conclusiones en torno al juego nacen de la experiencia adquirida en diferentes acciones desarrolladas en el espacio público. Nuestras intervenciones invitan al ciudadano a conocer las tradiciones y la cultura local propias del lugar a través del juego, así como entenderlas dentro del contexto global actual. Las propuestas contienen dos capas, independientes pero intrínsecamente relacionadas: la capa física, que permite disfrutar del juego con los recursos existentes, y la digital, que aumenta la experiencia de juego mediante la tecnología. El diseño del juego en sí se plantea desde la filosofía del código abierto. De esta manera, adquiere la capacidad para ser replicado, modificado y ampliado por el usuario. 


\subsection{Ugarte Flota. The augmented playground}

Un juego, dos ciudades costeras y un objetivo: hundir la flota. Ugarte Flota suma a la reivindicación del espacio público como plataforma de juego la idea de aumentar la experiencia a través de la tecnología y la de conectar dos lugares físicamente distantes a través del juego. De esta forma, la acción se convierte en un augmented playground, un espacio de juego que aumenta su potencial, gracias a la incorporación de la capa digital (De Luca \& Bertolo, 2012).

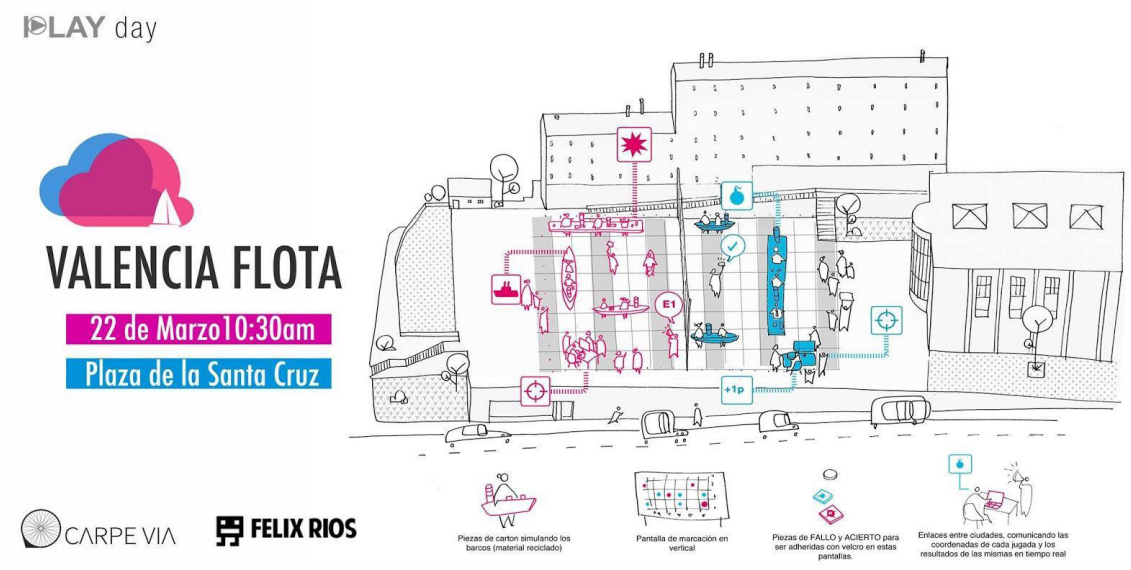

Figura 4. Panel de presentación de la propuesta Ugarte Flota. Carpe Via y Félix Rios. 2014

Esta propuesta, desarrollada en colaboración con Félix Ríos, se realizó en Play Day, una jornada de juegos urbanos en Otxarkoaga, Bilbao (Figura 4). El juego consistía en conectar un tablero a escala urbana en Bilbao con otro en Valencia para jugar a Hundir la flota. Los jugadores de cada ciudad, quienes adoptaban el rol de nave portando disfraces manufacturados, enviaban sus órdenes de ataque mediante tecnología móvil y streaming. Ugarte Flota buscaba conectar dos contextos locales de una manera global; este juego fue creado con el objetivo del disfrute, y sirvió como herramienta para el conocimiento de la cultura de la otra comunidad.

\subsection{Xarranca Rec. La reinvención del juego popular}

Xarranca Rec, propuesta ganadora de Rec Art Performance, fue llevada a cabo en el Rec09, un festival de moda en espacios industriales del barrio del Rec, Igualada. Dicho juego se desarrolló en todo el barrio, con el objetivo de generar un recorrido que re-descubriese un barrio deshabitado y olvidado, creando un diálogo entre diferentes elementos urbanos: bancos, fuentes y alcantarillas.

Xarranca Rec recupera un juego popular, perteneciente al imaginario colectivo, la rayuela, para aplicarlo a escala urbana en el espacio público. Éste genera una reacción automática en el usuario, una llamada a ser jugado, pues es intuitivo, no precisa instrucciones, abarca varias generaciones de jugadores y se puede jugar sin la necesidad de una coordinación compleja. 
Asímismo, permitía conocer la cultura local del lugar donde se desarrolló de dos formas: una a través de la tecnología mediante preguntas y desafíos con códigos $\mathrm{QR}$, y otra mediante el recorrido físico, revelando y conectando distintos espacios característicos y específicos del lugar.

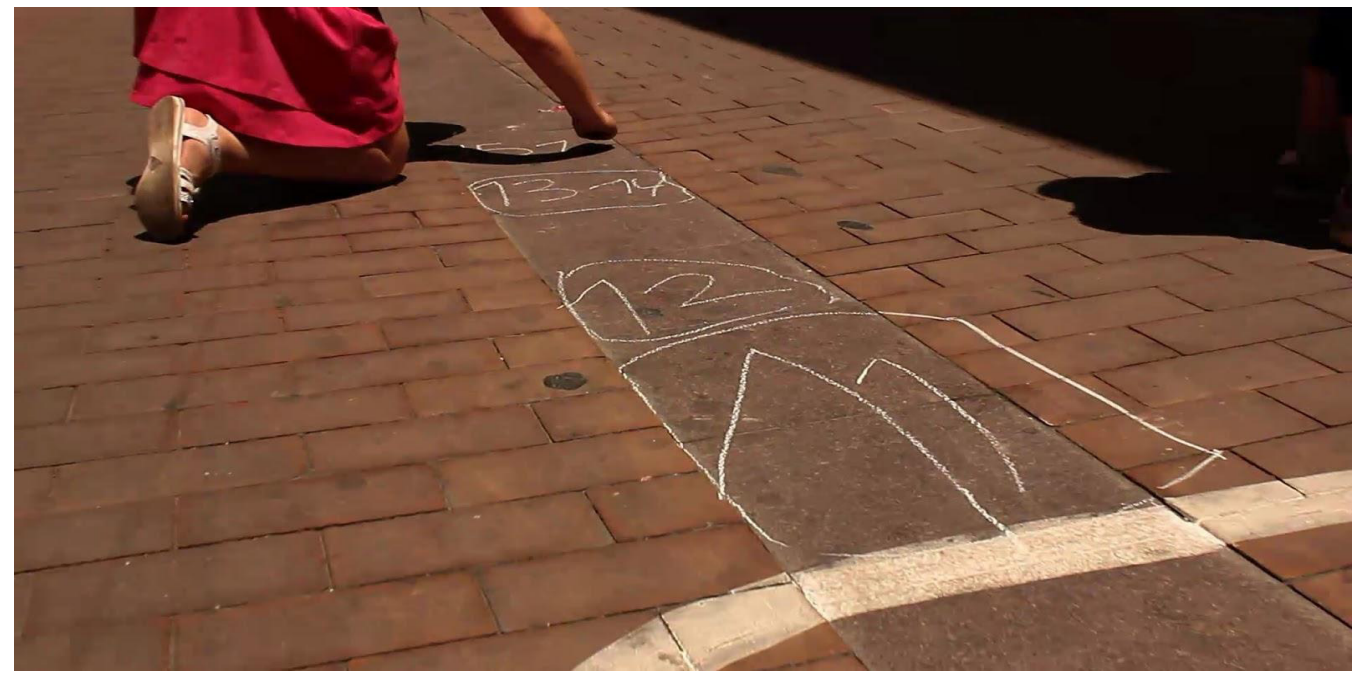

Figura 5. Ampliación de la Xarranca Rec por parte de los jugadores. Carpe Via. 2014.

Los códigos QR incluidos en el juego estaban situados en hitos y edificios destacados por su interés histórico o por formar parte de la memoria del lugar. Dichos códigos respondían a preguntas y retos formulados al jugador a lo largo del recorrido, que le animaban a conocer mejor el lugar. Otro rasgo característico de Xarranca Rec es su diseño, el hecho de que el jugador pueda replicar y ampliar el juego dónde y cuándo quiera (Figura 5). Un pack de juego gratuito y disponible en puntos estratégicos del recorrido, con una piedra y unas tizas, permite al usuario generar más xarranca, convertirse en creador espontáneo que puede ampliar y modificar el diseño inicial.

Si Xarranca Rec fue el origen de las experiencias propias con el juego popular como objeto, las siguientes acciones son la evolución de dicha propuesta. Comparten objetivos, pero desarrollan, a veces de manera fortuita, cometidos específicos.

En el caso del Tejetejo, desarrollado en el segundo certamen de activación sociocultural El Casc, en Villena, el juego fomentó la interacción social. A lo largo del recorrido -que subía desde la parte baja de la ciudad hasta las calles aparentemente más conflictivas de la parte alta- niños y mayores de diferentes etnias y grupos sociales salían y entraban de su recorrido habitual siguiendo el juego de manera espontánea. El juego se convirtió en herramienta de cohesión social. 


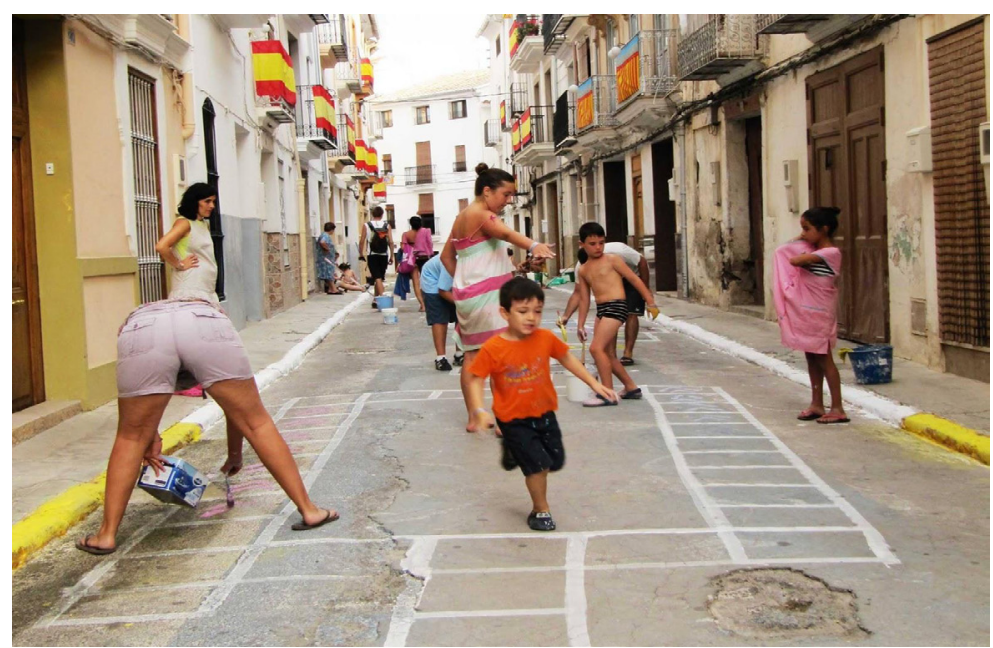

Figura 6. Gente pintando en la calle en Chelva. Carpe Via. 2014

En el Sambori de Maria Antonia Clavel los condicionantes desde los que se desarrolló la rayuela son muy particulares. Fue la primera acción fuera de un marco específico (festivales, certámenes o jornadas). El equipo de Carpe Via y los vecinos tomaron la iniciativa y. Así, el juego se convirtió en una actividad espontánea, no justificada por acontecimientos particulares, sino por el simple hecho de disfrutar.

Tradicionalmente, en la localidad de Chelva las calles se engalanan antes de las fiestas locales pintando diferentes motivos en el pavimento, salvo en esta ocasión en la que por diferentes motivos no era posible. Propusimos, pues, engalanar la calle mediante el juego: realizar una rayuela con la colaboración de los vecinos. De este modo, la elaboración y el diseño del juego no aterriza desde fuera en el entorno, sino que son los propios vecinos los autores del juego, y el equipo de Carpe Via se convierte en facilitadores que asesoran en el uso del material (Figura 6).

\subsection{Play the street! Manual de juegos urbanos.}

El pasado mes de octubre desarrollamos una propuesta para el concurso internacional de diseño social Hans Sauer Award 2015 basada en el conocimiento libre del juego urbano y su I+I+D colaborativo (Figura 7). Nuestro proyecto se centra en una plataforma física y virtual que procesa, almacena, investiga y fomenta el juego urbano en todas sus modalidades (tradicionales, innovadores, híbridos físico-digitales, locales, globales, etc.).

Play the street! Manual de juegos urbanos es un contenedor útil de conocimiento abierto y accesible para todo el mundo, donde la calle se convierte en un laboratorio urbano. Paralelamente, una vez ya experimentado, funciona como plataforma para el intercambio de experiencias y nuevos conocimientos sobre el juego. También permite mediante un mapeo -vía $A P P$ o vía web- detectar otros jugadores o juegos en tiempo real, contactar con ellos e iniciar un juego compartido en el espacio público. 
Así, este proyecto se convierte en una herramienta con un soporte físico a modo de libro y cuaderno con la calle como tablero y un soporte digital a modo de web y $A P P$, que sirve para ludificar y revitalizar el espacio público, proteger la memoria histórica del lugar fomentando su identidad local y devolver a la ciudad un carácter más dinámico, más divertido y más humano.
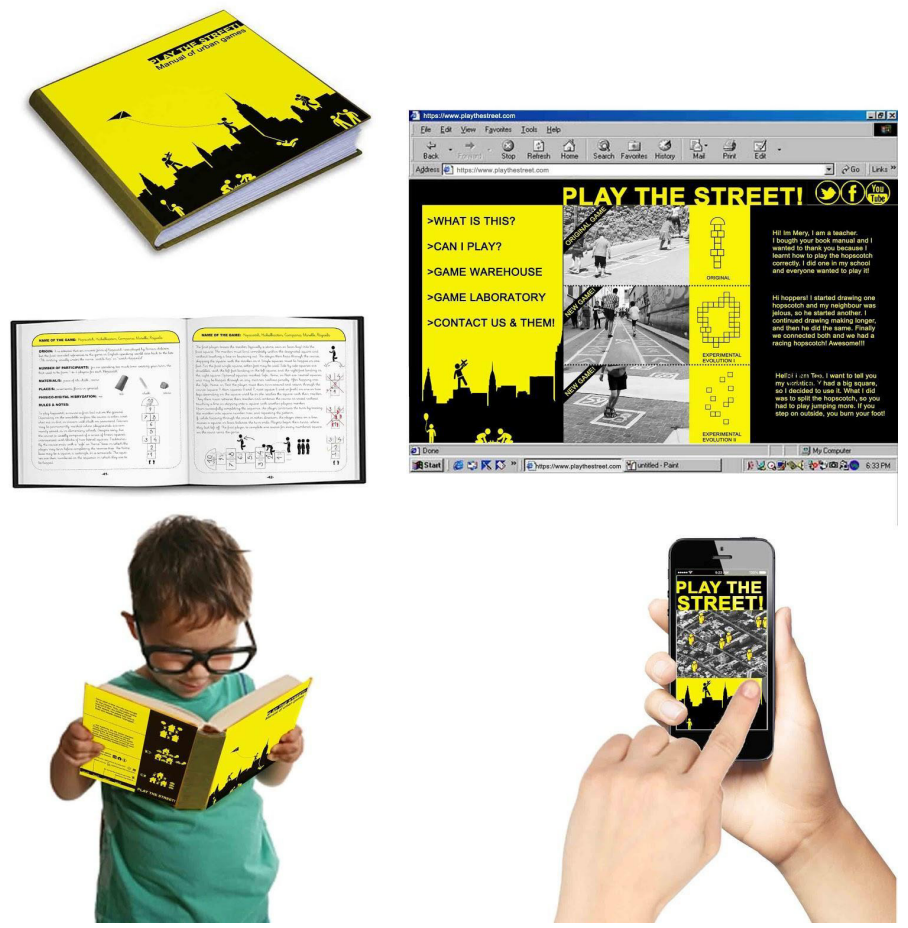

Figura 7. Play the Street! Manual de juegos urbanos. Carpe Via. 2014

\section{FUTURO: LA CIUDAD DE LA LUDOCRACIA}

Entendemos el término ludocracia como "la capacidad de mutación que conlleva un intercambio de la era del espectáculo (Debord, 2002) por la del simulacro lúdico (Gianetti, 2001)" (Mañas \& Pastor, 2014; p.13) y que "no apunta a una representación continuada y sin correlato en la realidad (Baudrillard, 1993) en un universo disneyficado, sino más bien a la posibilidad de generar una situación de contraste y resistencia frente al mero conformismo" (Mañas \& Pastor, 2014; p. 13).

Esta resistencia debe ser motivada por acciones que muestren que en el espacio público físico se pueden desarrollar las experiencias que hoy en día se han visto relegadas al mundo virtual. $A$ través del simulacro lúdico podremos ensayar diferentes soluciones que fomenten el desarrollo de la ciudad con un mayor consenso social para encontrar esa ciudad sin miedo a lo imprevisto, a lo no planeado o a la sorpresa, que se divierta en la diferencia, que se reinvente a cada segundo. Una ciudad que se entienda, de hecho, como un enorme tablero de juego. 
Por último, debemos deshacernos del aislamiento, del miedo a hacer, a jugar, al que estamos sometidos. Pasar de una ciudad con excesivas normas y restricciones -la ciudad del miedo- a una ciudad más sensible, capaz de adaptarse a las necesidades de todos los colectivos. En esta labor, el juego, la tecnología y la memoria juegan un papel fundamental: la tecnología, como amplificadora del espacio público; la memoria, como anhelo de recuperar la ciudad perdida, y el juego, como hecho inherente a la naturaleza humana.

\section{Referencias}

Baber, B., Karant, J., Küng, M., De Muynck, B., Pimlott, M., Voorthuis, J. (2005). Fear \& Space. The view of young designers in the Netherlands. Róterdam: Urban Affairs.

Basar, D., Miessen, M. (2009). ¿Alguien dijo participar? Un atlas de prácticas espaciales. Barcelona: Dpr-Barcelona.

Baudrillard, Jean. (1993). Cultura y simulacro. Barcelona: Kairós

Benedikt, M. (1993). Ciberespacio: Los primeros pasos. Consejo Nacional de Ciencia y Tecnología México. México: Equipo Sirius Mexicana.

Borja, J. (1998). Ciudadanía y espacio público. Urbanitats no 7: Ciutat real, ciutat ideal. Significat i funció a l'espai urbà modern, CCCB, Barcelona.

Castells, M. (2000). La era de la información: economía, sociedad y cultura. Volumen I, La sociedad en red. Madrid: Alianza.

Corsín Jiménez, A. (2014). The right to infrastructure: a prototype for open-source urbanism. Environment and Planning D: Society and Space, 32 (2) [html]. http://digital.csic.es/ bitstream/10261/85115/1/right infrastructure finalpreprint.pdf

De Luca, V., Bertolo, M. (2012). Urban Games to Design the Augmented City. Eludamos. Journal for Computer Game Culture. 2012; 6 (1), pp. 71-83 [html]. http://www.eludamos.org/index. php/eludamos/article/viewArticle/vol6no1-7/6-1-7-html

Delgado, M. (2011) En busca el espacio perdido. Prólogo del libro Territorios de la infancia: diálogos entre arquitectura y pedagogía [html]. http://manueldelgadoruiz.blogspot.com.es/2011/11/enbusca-del-espacio-perdido-prologo.html 
Deterding, S., Khaled, R., Nacke, L.E., Dixon, D., (2011) Gamification: Toward a Definition [html]. http://gamificationresearch.org/wp-content/uploads/2011/04/02-DeterdingKhaled-Nacke-Dixon.pdf>

Debord, Guy. (2002). La sociedad del espectáculo. Valencia: PreTextos

Di Siena, D. (2009). Espacios Sensibles. Departamento de Urbanística y Ordenación del Territorio de la Escuela Técnica Superior de Arquitectura de Madrid (UPM) [html]. http:// urbanohumano.org/castellano/espacios-sensibles-hibridacionfisico-digital-para-la-revitalizacion-de-los-espacios-publicos/>

Fratarelli, N. (2011). La ciudad mediática: El espacio público como amenaza. Revista Contratiempo nํ․ Arte, Pensamiento y Política [html]. http://www.revistacontratiempo.com.ar/ fratarelliciudad mediatica.pdf

Gianetti, C. (2001) Reflexiones acerca de la crisis de la imagen técnica, la interfaz y el juego. Anàlisi 27: 151-158

Harvey, D. (2013). Ciudades rebeldes: Del derecho de la ciudad a la revolución urbana. Salamanka: Akal.

Infans' Fans (2014). No hace falta nada, todo es necesario. La Ciudad Viva blog [html]. http://www.laciudadviva.org/ $\underline{\text { blogs } / ? p=21871}$

Lévy, P. (2004). Ciberdemocracia. Barcelona: UOC. Serie Nuevas Tecnologías y sociedad.

Mañas, M., Pastor, M. (2014). Acontecimientos, torpezas y derivas: generadores ludocráticos. AusArt Journal for Research in Art. vol.2 no1, p. 11-21 [html]. http://www.ehu.es/ojs/index.php/ ausart/arthicle/view/11983

Navazo, M. (2013). Placemaking: ¿y esto de qué va? (I). La Ciudad Viva blog [html]. http://www.laciudadviva.org/blogs/?p=18170

Punset, E. (2012) Redes. ¿Porquélos niñosseaburren en la escuela? [html]. https://www.youtube.com/watch?v=U2ql8CZwjws\#t=51

Reyes, J. (2013). La ciudad interfaz: Nuevas formas de regeneración urbana en código abierto. Valencia; Diazotec [html]. http://offlinecity.files.wordpress.com/2014/01/ciudadinterfaz-estrategias-de-regeneracic $3 b 3 n$-urbana-para-unaciudad-opensource-jonathan-reyes.pdf 
Ríos, F. (2014). Helium. Estrategias lúdicas de discusión y producción documental. Trabajo de Fin de Máster. Universidad Politécnica de Valencia

Sánchez Uzábal, A. (2009) Entrevista de Domenico di Siena a Alfonso Sánchez Uzábal [hmtl]. http://urbanohumano.org/ castellano/sentient-city-entrevista-a-alfonso-sanchez-skotperez/

Shepard, M. (2011). Sentient City: ubiquitous computing, architecture and the future of public space. Nueva York: MIT Press [html].

https://mitpress.mit.edu/books/sentient-city

Tonucci, F. (2002). Cuando los niños dicen: iBasta! Buenos Aires: Editorial Losada. S.A.

UNICEF (2004). Deporte, recreación y juego [hmtl]. http://www.unicef.org/spanish/adolescence/files/5571 SPORT $\underline{\text { SP.pdf }}$ 
